\title{
Physical activity for smoking cessation in pregnancy: randomised controlled trial
}

\author{
Michael Ussher, ${ }^{1}$ Sarah Lewis, ${ }^{2}$ Paul Aveyard, ${ }^{3}$ Isaac Manyonda, ${ }^{4}$ Robert West, ${ }^{5}$ Beth Lewis, ${ }^{6}$ \\ Bess Marcus, ${ }^{7}$ Muhammad Riaz, ${ }^{1}$ Adrian Taylor, ${ }^{8}$ Amanda Daley, ${ }^{9}$ Tim Coleman ${ }^{10}$
}

1Population Health Research Institute, St George's University of London, London SW17 ORE, UK 2Division of Epidemiology and Public Health and UK Centre for Tobacco and Alcohol Studies, University of Nottingham, Nottingham, UK

${ }^{3}$ Nuffield Department of Primary Care Health Sciences, University of Oxford, Oxford, UK

${ }^{4}$ Department of Obstetrics and Gynaecology, St George's University of London and St George's NHS Trust, London, UK

${ }^{5}$ Health Behaviour Research Centre, Department of

Epidemiology and Public Health, University College

London, London, UK

${ }^{6}$ School of Kinesiology, University of Minnesota, Minneapolis, MN, USA

${ }^{7}$ Department of Family and Preventive Medicine, University of California, San Diego, CA, USA 8Plymouth University Peninsula Schools of Medicine and Dentistry, Plymouth, Devon, UK

${ }^{9}$ Primary Care Clinical Sciences, University of Birmingham,

Birmingham, UK

${ }^{10}$ Division of Primary Care and UK Centre for Tobacco and Alcohol Studies, University of Nottingham, Nottingham, UK

Correspondence to: M Ussher mussher@sgul.ac.uk

Additional material is published online only. To view please visit the journal online (http://dx.doi. org/10.1136/bmj.h2145)

Cite this as: $B M J$ 2015;350:h2145 doi: 10.1136/bmj.h2145

Accepted: 23 March 2015

\section{ABSTRACT}

OBJECTIVE

To determine the effectiveness of a physical activity intervention for smoking cessation during pregnancy. DESIGN

Parallel group, randomised controlled, multicentre trial.

\section{SETTING}

13 hospitals in England, April 2009 to January 2014.

\section{PARTICIPANTS}

789 pregnant smokers, aged $16-50$ years and at $10-24$ weeks' gestation, who smoked at least one cigarette daily and were prepared to quit smoking one week after enrollment were randomised (1:1); 785 were included in the intention to treat analyses, with 392 assigned to the physical activity group.

\section{INTERVENTIONS}

Interventions began one week before a target quit date. Participants were randomised to six weekly sessions of behavioural support for smoking cessation (control) or to this support plus 14 sessions combining supervised treadmill exercise and physical activity consultations.

\section{MAIN OUTCOME MEASURES}

The primary outcome was continuous smoking abstinence from the target quit date until end of pregnancy, validated by exhaled carbon monoxide or salivary cotinine levels. To assess adherence, levels of moderate-vigorous intensity physical activity were self reported and in a $11.5 \%(n=90)$ random subsample of participants, physical activity was objectively measured by an accelerometer.

\section{RESULTS}

No significant difference was found in rates of smoking abstinence at end of pregnancy between the physical activity and control groups ( $8 \% \vee 6 \%$; odds ratio 1.21 , $95 \%$ confidence interval 0.70 to 2.10 ). For the physical

\section{WHAT IS ALREADY KNOWN ON THIS TOPIC}

The evidence for physical activity programmes aiding smoking cessation is mixed

Pregnancy provides a compelling rationale for their use as drugs are

contraindicated or ineffective and exercise has been shown to reduce cigarette

cravings among pregnant smokers

Until now a randomised controlled trial of physical activity for smoking cessation in pregnancy has been lacking

\section{WHAT THIS STUDY ADDS}

Our study suggests that a physical activity intervention has no benefit for helping women to quit smoking during pregnancy

However, physical activity remains indicated for general health benefits in pregnancy

activity group compared with the control group, there was a $40 \%$ (95\% confidence interval $13 \%$ to $73 \%$ ), $34 \%$ (6\% to $69 \%$ ), and $46 \%$ (12\% to $91 \%$ ) greater increase in self reported minutes carrying out physical activity per week from baseline to one week, four weeks, and six weeks post-quit day, respectively. According to the accelerometer data there was no significant difference in physical activity levels between the groups. Participants attended a median of four treatment sessions in the intervention group and three in the control group. Adverse events and birth outcomes were similar between the two groups, except for significantly more caesarean births in the control group than in the physical activity group ( $29 \%$ v $21 \%$, $\mathrm{P}=0.023)$.

\section{CONCLUSION}

Adding a physical activity intervention to behavioural smoking cessation support for pregnant women did not increase cessation rates at end of pregnancy. During pregnancy, physical activity is not recommended for smoking cessation but remains indicated for general health benefits.

\section{TRIAL REGISTRATION \\ Current Controlled Trials ISRCTN48600346.}

\section{Introduction}

Maternal smoking in pregnancy is the main preventable cause of morbidity and death among women and infants. Smoking is associated with adverse pregnancy and birth outcomes, including miscarriage, still birth, prematurity, low birth weight, congenital abnormalities, and neonatal or sudden infant death. ${ }^{1-3}$ In most high income countries at least $10 \%$ of women smoke during pregnancy, ${ }^{4-6}$ and the prevalence in low and middle income countries is rising. ${ }^{7}$ Smoking cessation during pregnancy improves birth outcomes, ${ }^{8}$ and behavioural support helps pregnant women to stop smoking. ${ }^{8}$ Use of nicotine replacement therapy during pregnancy seems to have little or no effect, and other pharmaceutical aids are contraindicated. ${ }^{9}$ The majority of pregnant women who receive behavioural support for smoking cessation do not stop ${ }^{8}$; thus new interventions that add to the effectiveness of behavioural support are needed.

Physical activity programmes may add to the effectiveness of behavioural support. In non-pregnant smokers there is convincing evidence that physical activity reduces the intensity of urges to smoke, which are the main cause of relapse to smoking. ${ }^{10}$ Again in nonpregnant smokers, the trial evidence that physical activity aids cessation is mixed, with only one trial-of vigorous intensity physical activity-showing a long term benefit, ${ }^{11}$ although most trials were small and had other design 
features that made the evidence difficult to interpret. ${ }^{12}$ Adequately powered trials are needed with moderate intensity exercise (for example, brisk walking), which is likely to be more acceptable than vigorous exercise for most people. ${ }^{13}$ Moderate intensity physical activity is recommended for pregnancy, and pregnant smokers are likely to be receptive to such an intervention. ${ }^{14}$ We conducted a parallel group, randomised controlled trial to assess the effectiveness of a physical activity intervention for smoking cessation during pregnancy.

\section{Methods}

\section{Study population}

Between April 2009 and November 2012, we recruited pregnant women by telephone after their first antenatal booking visit (conducted at either hospital or a community clinic), from 13 hospital antenatal clinics in London, Surrey, Kent, and Cheshire. In the United Kingdom all pregnant women are booked for delivery in the secondary care setting, although a small fraction of women deliver at home or in primary care (midwife led) units. Pregnant smokers are, by definition, high risk and would be expected to be cared for in hospital. Inclusion criteria were wanting to stop smoking, wanting help with stopping smoking, agreeing to set a date for quitting smoking within one week of the baseline visit, age 16-50 years, being at 10-24 weeks of gestation, cigarette consumption of five or more daily before pregnancy, currently smoking one or more cigarettes daily, and being able to walk continuously for 15 minutes. Exclusion criteria were medical conditions potentially exacerbated by exercise or advised against exercise by a doctor, inability to provide informed consent or complete questionnaires in English, drug or alcohol dependence, and currently using or wanting to use nicotine replacement therapy. We recruited women irrespective of their current level of physical activity or motivation towards increasing their activity.

\section{Study protocol and interventions}

Wandsworth research ethics committee approved the published protocol ${ }^{15}$ (available at www.trialsjournal. com/content/13/1/186). All participants provided written informed consent. We offered all participants six weekly sessions of 20 minutes of individual behavioural cessation support, starting one week before the quit date and ending four weeks afterwards. This intervention aimed to support smoking cessation by reinforcing commitment to abstinence and solving women's problems about maintaining abstinence. It incorporated all 43 behaviour change techniques defined in a published taxonomy ${ }^{16}$ and as described in the protocol, ${ }^{15}$ except for the provision of rewards contingent on successfully stopping smoking. The emphasis was on the importance of avoiding lapses, managing withdrawal symptoms and urges to smoke, enhancing self confidence, and prevention of relapse.

At enrollment we randomised participants to behavioural cessation support alone or to behavioural cessation support plus a physical activity intervention, combining supervised exercise with physical activity consultations. Fourteen sessions of supervised exercise were offered over eight weeks; twice a week for six weeks then weekly for two weeks. At each session the participants walked at a moderate intensity on a treadmill for up to 30 minutes. Immediately before each treadmill session, the women received behavioural support. At the first two treadmill sessions, and then on every other occasion (total of nine consultations), this support aimed to identify opportunities to incorporate physical activity into women's lives, to motivate them to use physical activity to reduce the urge to smoke, and to help them use behavioural strategies to improve adherence to these plans. These 20 minute consultations incorporated 19 behaviour change techniques, as described in the study protocol. ${ }^{15}$ The women were advised to be active for at least 10 minutes at a time, progressing towards 30 minutes of activity on at least five days a week. The emphasis was on brisk walking, and pedometers (Digi-Walker SW-200; Yamax, Nottingham, UK) were supplied, with researchers setting individualised step count targets. We also provided a DVD on antenatal exercise. On the other occasion the women received behavioural support for smoking sessions (up to six sessions) as for the control group. For each session attended, participants were paid $€ 7$ towards travel.

\section{Randomisation}

An independent statistician generated a randomisation list using Stata, with random permuted blocks of random size stratified by recruitment centre, in a 1:1 ratio. At enrollment the sequence was concealed from researchers who confirmed consent and eligibility on an online database before allocation was revealed. It was not feasible to mask participants or researchers to group allocation.

\section{Data collection}

We collected personal and smoking characteristics of the women at baseline, including score on the Fagerström test for cigarette dependence, ${ }^{17}$ self reports of moderate-vigorous intensity physical activity in the previous week (bouts of $\geq 10$ minutes) using the seven day physical activity recall interview, ${ }^{18}$ Edinburgh postnatal depression scale score, ${ }^{19}$ confidence about taking up physical activity ${ }^{20}$ and stopping smoking, ${ }^{21}$ alcohol consumption, ${ }^{22}$ weekly cigarette withdrawal symptoms, ${ }^{23}$ and weekly smoking urges (combining ratings of strength and frequency). ${ }^{24}$ At the first antenatal booking visit the midwife measured the women's clothed weight (without shoes) on a digital scale. During all contacts, the women were asked about adverse events. Research midwives examined the women's medical records monthly for adverse events. The records were also checked by the research midwives for maternal and infant outcome data after delivery.

\section{Outcomes}

The primary outcome was self reported continuous abstinence from smoking between quit date and end of pregnancy. We permitted temporary, brief smoking 
lapses of up to five cigarettes in total (on up to five occasions). ${ }^{25}$ Abstinence was validated by the concentration of either exhaled carbon monoxide $(<8 \mathrm{ppm})$ or salivary cotinine $(<10 \mathrm{ng} / \mathrm{mL})$; if both measures were available both were required for validation. We assessed the levels of carbon monoxide weekly up to four weeks after the quit day and at the end of pregnancy. Cotinine was measured at four weeks post-quit date and end of pregnancy.

Secondary outcomes included validated abstinence at four weeks after the quit date and self reported abstinence by telephone at the final follow-up at six months after birth (completed in January 2014). At one week after the quit date we further assessed weekly urges to smoke, weekly withdrawal symptoms, and confidence about taking up physical activity and stopping smoking. We also further assessed the Edinburgh postnatal depression scale scores at the end of pregnancy and six months after birth. A researcher weighed the women at the end of pregnancy using the same method as used by the midwife at the first antenatal booking visit.

To assess adherence we assessed self reported physical activity at weeks 1, 4, and 6 after the quit date and at the end of pregnancy. In an $11.5 \%$ random subsample of participants (the target was 10\%) physical activity was objectively measured using an accelerometer (Model GT1M or GT3X; Actigraph, Pensacola, FL). Only 90 women were asked to wear an accelerometer as our pilot work showed that most women would not tolerate devices worn around the waist, and at the start of the study validated wrist worn accelerometers were not commercially available; therefore, as the only practicable alternative, we used self reported physical activity for the primary analysis of physical activity. The accelerometer was worn over the right hip, in the fourth week after the quit date and for up to seven consecutive days, recording "dry land" activity during waking hours at one minute epochs. Use of National Health Service behavioural support for smoking cessation, beyond that offered in the study, and nicotine replacement therapy were self reported. Participants received a £10 shopping voucher for completing end of pregnancy and six month postnatal follow-ups (that is, £20 maximum).

\section{Statistical analysis}

We anticipated a cessation rate of $15 \%$ in the control group, on the basis that $9 \%$ of pregnant women who are smokers stop smoking with usual care after their first antenatal visit, and that with behavioural support another $6-7 \%$ quit. ${ }^{26}$ Based on pilot work, ${ }^{14}$ we projected a cessation rate of $23 \%$ in the treatment group. We calculated that 866 participants would provide $83 \%$ power at a 5\% significance level to detect an absolute difference of 8 percentage points in the occurrence of the primary outcome between the two groups (that is, $15 \%$ in the control group versus $23 \%$ in the physical activity group), corresponding to an odds ratio of 1.69 or a relative risk of 1.53 . The prime aim of assisting smoking cessation in pregnancy is to improve the outcome of the pregnancy. The latest version of the Cochrane review of psychosocial interventions for supporting women to stop smoking in pregnancy shows evidence that such interventions were effective in helping women stop smoking and improving perinatal outcomes. ${ }^{27}$ For example, the main subgroup in the review comprising counselling versus usual care showed a relative risk of 1.44 for achieving abstinence in late pregnancy, the same outcome we used. No individual trial of smoking cessation in pregnancy detected differences in perinatal outcomes by intervention status, but evidence of this has been shown by the meta-analysis of all trials in the review. When pooled together, these interventions produced the following relative risks for intervention versus control: low birth weight 0.82 (95\% confidence interval 0.71 to 0.94 ), preterm birth 0.82 (0.70 to 0.96 ), and increased mean birth weight $41 \mathrm{~g}$ (18 to $63 \mathrm{~g}$ ). Thus, relative increases in the rate of cessation of a similar size to the one we were aiming to detect have led to meaningful improvements in perinatal outcomes and would be expected to do so in this trial.

Analysis was on an intention to treat basis; we assumed participants with missing outcome data to be smoking. ${ }^{25}$ Using logistic regression we compared the proportion of women reporting continuous smoking abstinence at the end of pregnancy between study groups, with adjustment for recruitment centre. We assessed statistical significance with the likelihood ratio test, with the estimate of effect given as the odds ratio and 95\% confidence interval. A secondary analysis adjusted for recruitment centre and the potentially important prognostic baseline factors of cigarette dependence, age, depression, maternal educational level, and partner's smoking status. In addition, we tested for an interaction between baseline physical activity ( $<150$ mins/week moderate-vigorous physical activity versus $\geq 150$ mins/week moderate-vigorous physical activity) and the rates of smoking abstinence at the end of pregnancy. We also assessed whether changes in physical activity levels affected smoking cessation rates: exclusively in the physical activity group we assessed, among those reporting < 150 mins/week moderate-vigorous physical activity at baseline, whether reporting $\geq 150$ mins/week moderate-vigorous physical activity at four weeks or six weeks after the quit day was associated with smoking abstinence at those times.

For the primary outcome, to assess the influence of the assumption that missing data equals "smoking" on the effect size, we tested various scenarios of the association between smoking and having missing data using the Hedeker method. ${ }^{28}$ For ratings of withdrawal symptoms, urge to smoke, confidence for quitting smoking, and confidence for taking physical activity we calculated a change score between baseline and one week post-quit, and we used $t$ tests to compare scores for the two groups. We compared the use of nicotine replacement therapy and behavioural support between the two groups using $\chi^{2}$ tests.

We $\log$ transformed (log base 10) self reported weekly minutes of moderate-vigorous physical activity (in bouts of $\geq 10$ minutes) to normality, and we analysed the difference in self reported physical activity 
between the groups over time using a mixed effects linear regression model to account for within person correlations over time. In this model, we estimated the difference between treatment groups at each time point with adjustment for visit time, baseline minutes of moderate-vigorous physical activity, the interaction of visit time and baseline minutes of moderate-vigorous physical activity, and recruitment centre. We analysed the accelerometer data using KineSoft software (version 3.3.76; Loughborough, UK). Files with at least 10 hours of valid wear time on one or more days were retained in the analyses. We used standard cut-off points to determine moderate-vigorous physical activity. ${ }^{29}$ The two study groups were compared (Mann-Whitney tests) for accelerometer reports of moderate-vigorous physical activity, both when restricting the analysis to bouts of at least 10 minutes (to allow comparison with the self report data) and when including all moderate-vigorous physical activity, irrespective of duration. For the week when the accelerometer was worn we used Wilcoxon tests to compare self reports of moderate-vigorous physical activity with accelerometer data.

We compared the changes in depression scores for the two groups using a mixed effect linear regression, adjusted for visit time, baseline Edinburgh postnatal depression scale score, the interaction of visit time and baseline Edinburgh postnatal depression scale score, and recruitment centre. This model allows for correlation between the repeated measurements at the end of pregnancy and six months. Maternal weight at end of pregnancy was compared for the two groups using linear regression, with adjustment for early pregnancy weight and recruitment centre. As some of the "end of pregnancy" weights were recorded before the birth and some after, we conducted separate regressions for

\section{Women recorded as smokers at first antenatal visit $(n=8096)$}

Excluded $(n=7307)$

Unable to contact $(n=2169)$

Declined to participate $(n=2324)$

Did not meet inclusion criteria $(n=2583)$

Other reasons $(n=231)$

Randomisation $(\mathrm{n}=789)$

\section{$\downarrow$}

Assigned to behavioural support plus physical activity intervention $(n=394)$

Received behavioural support plus physical activity intervention $(n=393)$

\section{Lost to follow-up $(n=35)$ :}

Lost to follow-up $(n=35)$

Fetal or infant deaths $(n=9)$

Withdrew consent before providing baseline

data $(n=1)$

Unable to contact or refused to attend $(n=25)$ $\downarrow$

Included in primary analysis $(\mathrm{n}=392)$

Excluded from analysis owing to being enrolled

twice $(n=2)$

Fig 1 | Flow of participants through trial. Participants lost to follow-up included women who experienced fetal or infant loss and were not assessed for smoking status
Included in primary analysis $(\mathrm{n}=393)$

Excluded from analysis as did not meet inclusion criteria at baseline visit $(n=2)$ before (gestational weight change) and after (postnatal weight retention) birth.

Using logistic regression or linear regression adjusted for recruitment centre we compared fetal and maternal birth outcomes between the two groups. For fetal outcomes, the primary analysis was of singleton births. We also conducted a sensitivity analysis, including multiple births, with clustering of outcomes accounted for by using an approach that regards each woman as the "cluster" and her number of offspring as the cluster size. ${ }^{30} \mathrm{~A} \chi^{2}$ test was used to compare adverse events for the groups.

\section{Results}

Figure 1 shows the numbers of participants who were enrolled and excluded. Four women were excluded post-randomisation; two women (physical activity group) were enrolled twice in sequential pregnancies (their second enrolment was removed) and two women (control group) were ineligible at their baseline visit and had been randomised erroneously. When ineligible patients are mistakenly randomised into a trial it is acceptable to exclude participants' data within an intention to treat approach without risking bias. ${ }^{31}$ of 785 pregnancies, 774 were singleton, 10 were twins, and one was unknown as the participant withdrew consent. The baseline characteristics of participants in the two groups were similar (table 1$)$. Over half $(54 \%)$ were smoking at least 10 cigarettes a day. By self report, 70\% were achieving the recommendation of 150 minutes a week of moderate-vigorous physical activity (in bouts of $\geq 10$ minutes). ${ }^{13}$

The follow-up rate for the primary outcome was $88.8 \%$ (697 participants), and this was similar for the two study groups. Of the 88 participants (11.2\%) who did not complete the assessments necessary for the analysis of the primary outcome, 43 (48.9\%) were known to have smoked from the follow-up assessments that they did complete. Evidence was lacking of a significant difference in this rate between study groups. Only for the remaining 45 participants (5.7\% of all participants) was it necessary to assume continued smoking or relapse.

Participants attended a median of four treatment sessions in the intervention group and three in the control group (table 2). Physical activity increased after baseline, but the increase was significantly greater in the physical activity group, by 33\% (95\% confidence interval $14 \%$ to $56 \%$ ), $28 \%$ (7\% to $52 \%$ ), and $36 \%$ (12\% to $65 \%$ ) at one week, four weeks, and six weeks post-quit day, respectively (fig 2, table 2). Only 28 women reported receiving NHS face to face support for smoking cessation, besides that offered in the trial, and 60 women reported using nicotine replacement therapy; the numbers reporting this support were similar between the two groups.

Of 90 participants ( $11.5 \%$ of total sample) asked to wear an accelerometer during the fourth week after the quit date, 78 (87\%) provided valid data (37 in physical activity group), 10 provided insufficient data, and two wore devices with technical problems. Participants providing accelerometer data had similar baseline characteristics 
Table 1 | Baseline characteristics, according to study group. Values are numbers (percentages) unless stated otherwise

\begin{tabular}{|c|c|c|}
\hline Characteristics & $\begin{array}{l}\text { Physical activity } \\
\text { group }(\mathrm{n}=391)\end{array}$ & $\begin{array}{l}\text { Control group } \\
(\mathrm{n}=393)\end{array}$ \\
\hline Mean (SD) age (years) & $27.2(6.1)$ & $27.8(6.5)$ \\
\hline Mean (SD) age at leaving full time education (years)* & $17.8(3.0)$ & $18.0(3.3)$ \\
\hline Mean (SD) weight $(\mathrm{kg}) \dagger$ & $69.2(14.1)$ & 70.9 (15.9) \\
\hline Mean (SD) body mass index $\left(\mathrm{kg} / \mathrm{m}^{2}\right) \dagger$ & $25.6(5.0)$ & $26.6(5.6)$ \\
\hline Mean (SD) gestational age (weeks) & $15.6(3.3)$ & $15.6(3.3)$ \\
\hline Mean (SD) Edinburgh postnatal depression scale score & $7.6(5.3)$ & $7.7(5.0)$ \\
\hline $\begin{array}{l}\text { Median (interquartile range) No of cigarettes smoked } \\
\text { daily before pregnancy }\end{array}$ & $20(12-20)$ & $20(12-20)$ \\
\hline $\begin{array}{l}\text { Median (interquartile range) No of cigarettes smoked } \\
\text { daily at randomisation }\end{array}$ & $10(5-12)$ & $10(5-15)$ \\
\hline $\begin{array}{l}\text { Median (interquartile range) Fagerström test of } \\
\text { cigarette dependence score } \neq\end{array}$ & $4(2-5)$ & $4(2-5)$ \\
\hline $\begin{array}{l}\text { Median (interquartile range) expired carbon monoxide } \\
\text { level (ppm)§ }\end{array}$ & $10(7-14)$ & $10(6-14)$ \\
\hline $\begin{array}{l}\text { Median (interquartile range) self report of weekly } \\
\text { moderate-vigorous physical activity (mins) }\end{array}$ & $210(125-350)$ & $225(130-360)$ \\
\hline Married or living with partner & $230(59)$ & $221(56)$ \\
\hline Partner smokes & $261(67)$ & $250(64)$ \\
\hline White ${ }^{\star \star}$ & $308(79)$ & $298(76)$ \\
\hline Professional or managerial occupation & $46(12)$ & $53(13)$ \\
\hline Smoked in a previous pregnancyt† & $186(78)$ & $193(78)$ \\
\hline Edinburgh postnatal depression scale score $\geq 13$ & $68(17)$ & $75(19)$ \\
\hline $\begin{array}{l}\text { Self report of } \geq 150 \text { mins/week of moderate-vigorous } \\
\text { physical activity }\end{array}$ & $275(70)$ & $273(69)$ \\
\hline Self report of walking as main type of physical activity & $301(77)$ & $313(80)$ \\
\hline \multicolumn{3}{|l|}{ Parity§§: } \\
\hline $0-1$ & $317(81)$ & $309(79)$ \\
\hline $2-3$ & $67(17)$ & $75(19)$ \\
\hline$>4$ & $7(2)$ & $9(2)$ \\
\hline Previous preterm birth $\neq \ddagger$ & $68(17)$ & $61(16)$ \\
\hline Very or extremely high confidence for quitting smoking & $89(23)$ & $98(25)$ \\
\hline $\begin{array}{l}\text { Very or extremely confident of doing } 30 \text { mins of physical } \\
\text { activity on at least } 5 \text { days a week during pregnancy }\end{array}$ & $274(70)$ & $277(71)$ \\
\hline Takes alcohol more than twice a week & $6(2)$ & $5(1)$ \\
\hline Consumes $>3$ alcoholic drinks on a drinking day $\|^{\star \star \star \star}$ & $14(16)$ & $3(4)$ \\
\hline
\end{tabular}

Baseline data were not recorded for one participant in physical activity group who withdrew consent shortly after randomisation.

${ }^{*}$ Data exclude 41 women who were still in full time education.

tNot recorded for one participant in control group.

$\ddagger$ Not recorded for one participant in physical activity group.

$\S$ Not recorded for one participants in physical activity group and two in control group.

IExcludes 92 women with no partner.

**Race or ethnic group was self reported and categorised according to standard UK census categories.

t†Excludes 297 with no previous pregnancies.

‡¥Any previous pregnancy of duration 24-37 weeks.

$\S \S$ Number of previous pregnancies progressing beyond 24 weeks.

११Excludes 617 women responding "not applicable" (that is, not consuming alcohol).

$\star \star \star S i g n i f i c a n t$ difference between groups ( $P=0.011)$, using Fisher's exact test. This variable was not related to the

primary outcome and therefore was not adjusted for in the analysis. There were no other significant differences

between the groups. (in bouts of $\geq 10$ minutes) was significantly higher by self report than by accelerometer; this being the case for the control group (median difference 23.2 (interquartile range 6.1-37.1) minutes, $\mathrm{P}<0.001$ ), and even more so for the physical activity group (29.7, 17.1-49.4) minutes, $\mathrm{P}<0.001)$.

There was no significant difference in smoking abstinence rates between the two groups at follow-up (table 3). The rate of validated continuous abstinence at end of pregnancy was $8 \%$ in the physical activity group and $6 \%$ in the control group (odds ratio for physical activity group, adjusted for recruitment centre only $1.21,95 \%$ confidence interval 0.70 to 2.10 ). At four weeks the validated abstinence rate was $13 \%$ in the physical activity group and 16\% in the control group (odds ratio adjusted for recruitment centre only 0.79 , 0.53 to 1.18). At six months after birth, the rate of self reported abstinence was $6 \%$ in the physical activity group and $4 \%$ in the control group (odds ratio adjusted for recruitment centre only 1.55, 95\% confidence interval 0.81 to 2.97). Fully adjusted analyses yielded similar findings (table 3). The sensitivity analyses showed that the observed effect size and its statistical significance were independent of the influence of missing data for the primary outcome. There was no significant interaction between baseline self reports of moderate-vigorous physical activity $(<150 v \geq 150 \mathrm{mins} /$ week physical activity) and the effect of the intervention on smoking abstinence at the end of pregnancy (adjusted for recruitment centre only: odds ratio 3.03, 95\% confidence interval 0.68 to $13.40, \mathrm{P}=0.129)$. Nor was there any evidence that changes in self reports of physical activity in the physical activity group were significantly associated with smoking abstinence (adjusted for recruitment centre only: odds ratio at four weeks 2.21 , $95 \%$ confidence interval 0.48 to $10.12, \mathrm{P}=0.321$, and at six weeks 2.36, 0.41 to $13.51, \mathrm{P}=0.362$ ).

At the end of pregnancy, the Edinburgh postnatal depression scale score was significantly higher $(\mathrm{P}=0.017)$ for the physical activity group compared with the control group (table 4). There was no significant difference in scores between the groups at six months. There was no significant difference in gestational or postnatal weight between the groups (table 5). The baseline characteristics among those providing data on depression and weight were similar to those for the total trial sample and were comparable between the study groups.

Between baseline and one week post-quit, ratings for confidence for participating in physical activity for the physical activity compared with control group increased significantly $(\mathrm{P}=0.004)$, but there was no significant change in ratings for individual withdrawal symptoms, urge to smoke, or confidence for quitting smoking. The total number of women or their infants who had at least one adverse event or serious adverse event was 217 (55\%) in the physical activity group and 219 (56\%) in the control group (odds ratio $0.99,95 \%$ confidence interval 0.75 to 1.32) (see supplementary table on bmj.com). There were no significant differences between the groups for any adverse event or serious adverse event, 


\begin{tabular}{|c|c|c|c|c|}
\hline Assessment time & Physical activity group & Control group & Relative change* $(95 \% \mathrm{Cl})$ & P value \\
\hline \multicolumn{5}{|c|}{ No, median (interquartile range) self reported weekly MVPA (mins) } \\
\hline Baseline & $391,210(125-350)$ & $393,225(130-360)$ & - & - \\
\hline \multicolumn{5}{|l|}{ No of weeks post-quit day: } \\
\hline 1 & $162,280(190-425)$ & $206,240(140-420)$ & $1.33(1.14$ to 1.56$)$ & $<0.001$ \\
\hline 4 & $135,270(180-420)$ & $157,210(120-340)$ & 1.28 (1.07 to 1.52$)$ & 0.006 \\
\hline 6 & $90,277(180-400)$ & $121,220(130-350)$ & $1.36(1.12$ to 1.65$)$ & 0.002 \\
\hline End of pregnancy & $188,155(100-240)$ & $187,140(60-240)$ & 1.25 (0.96 to 1.61$)$ & 0.093 \\
\hline 6 month follow-up & $147,180(80-330)$ & $136,135(60-285)$ & $1.16(0.85$ to 1.59$)$ & 0.339 \\
\hline \multicolumn{5}{|c|}{ No, mean (SD) time walked on treadmill during supervised exercise (mins) } \\
\hline Baseline & $390,12.2(7.5)$ & - & - & - \\
\hline \multicolumn{5}{|l|}{ No of weeks post-quit day: } \\
\hline 1 & $163,19.0(8.5)$ & - & - & - \\
\hline 4 & $134,15.2(10.8)$ & - & - & - \\
\hline 6 & $90,17.7(10.9)$ & - & - & - \\
\hline No of treatment sessions attended & $391,4(2-8) \dagger$ & $393,3(2-6)$ & & \\
\hline \multicolumn{5}{|c|}{$\begin{array}{l}\text { MVPA=moderate-vigorous physical activity. } \\
\text { *Relative change of physical activity mixed effect model for log of physical activity. Adjusted for visit time, baseline minutes of MVPA, interaction of visit } \\
\text { time and baseline minutes of MVPA, and recruitment centre. } \\
\text { tMedian (interquartile range) numbers of sessions attended for both physical activity counselling and smoking cessation behavioural support were } 2 \\
(1-4) \text {. Participants received supervised exercise at all sessions. }\end{array}$} \\
\hline
\end{tabular}

including maternal or fetal adverse events as probable complications of pregnancy or neonatal adverse events. Birth outcomes were similar between the two groups, including miscarriages, stillbirths, neonatal deaths, birth weights, and preterm births, except there were significantly fewer deliveries by caesarean section in the physical activity group than in the control group (29\% $\mathrm{v}$ $21 \%, \mathrm{P}=0.023$, table 6 ). When including twin births the findings for birth outcomes were similar.

\section{Discussion}

Supplementing behavioural support with a physical activity intervention was no more effective in aiding smoking cessation during pregnancy than behavioural support alone. These findings were observed despite women in the physical activity group reporting 33\% to $36 \%$ greater increases in physical activity than women in the control group during the intervention period.

\section{Strengths and limitations of this study}

In this study the limitations of previous trials of physical activity interventions for smoking cessation ${ }^{12}$ were

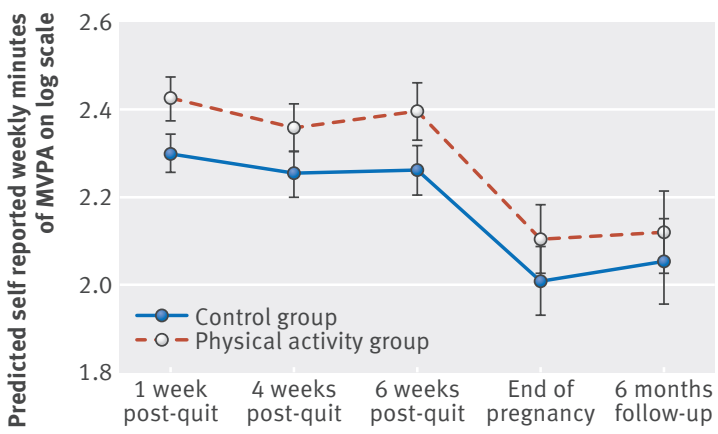

Visit time

Fig 2 Comparison of self reported levels of moderatevigorous physical activity (MVPA) in trial groups at different time points. Prediction on log scale from mixed effect model overcome by implementing a multicentre trial, offering an intensive physical activity intervention with support to increase physical activity as an aid for smoking cessation in addition to supervised exercise sessions, assessing and validating physical activity in both groups, using a robust outcome of continuous abstinence, and offering a pragmatic intervention that could be readily integrated into routine healthcare in the NHS. At baseline the participants' average gestational age, age, and cigarette consumption were similar to that of participants in another recent large pregnancy and smoking cessation trial conducted in the United Kingdom. ${ }^{32}$ The frequency of occurrence of adverse events and birth outcomes were also similar. These women were generally representative of women who smoke, ${ }^{8}$ and the findings are likely to be generalisable to primary and secondary care settings. The prevalence of probable depression at baseline is slightly higher than that reported for pregnant women in general. ${ }^{33}$

This study was approximately twice the size of previous randomised controlled trials investigating physical activity interventions for smoking cessation delivered through face to face support. ${ }^{12}$ As anticipated, we recruited around $10 \%$ of pregnant women recorded as smokers at their first antenatal booking visit. However, the prevalence of smoking was lower than anticipated; therefore, despite extending the recruitment period, our sample was only $91 \%$ of the target 866 . Quit rates were lower than anticipated in our power calculation, which will also have reduced the power of the study. The confidence intervals around the odds ratio for the effect of the intervention show the level of precision of this estimate, given the achieved sample size, and imply that we cannot rule out up to a twofold increase in abstinence at the end of pregnancy compared with controls at the upper end of the confidence interval, although our point estimate suggests an effect of intervention that is unlikely to be clinically meaningful. Additionally, at our four week outcome, quit rates were 


\begin{tabular}{|c|c|c|c|c|}
\hline Outcomes & $\begin{array}{l}\text { Physical activity group } \\
(\mathrm{n}=392)\end{array}$ & $\begin{array}{l}\text { Control group } \\
(\mathrm{n}=393)\end{array}$ & $\begin{array}{l}\text { Odds ratio* }(95 \% \mathrm{Cl}) \\
\text { with basic adjustment }\end{array}$ & $\begin{array}{l}\text { Fully adjusted odds } \\
\text { ratiot }(95 \% \mathrm{CI})\end{array}$ \\
\hline \multicolumn{5}{|l|}{ Primary: } \\
\hline $\begin{array}{l}\text { Self reported continuous abstinence } \neq \text { at end of pregnancy§ } \\
\text { with biochemical validation }\end{array}$ & $30(8)$ & $25(6)$ & 1.21 (0.70 to 2.10$)$ & 1.37 (0.78 to 2.41$)$ \\
\hline \multicolumn{5}{|l|}{ Secondary: } \\
\hline $\begin{array}{l}\text { Self reported continuous abstinence } 4 \text { weeks post-quit day, } \\
\text { with validation** }\end{array}$ & 50 (13) & $61(16)$ & 0.79 (0.53 to 1.18) & 0.87 (0.57 to 1.31) \\
\hline Self reported continuous abstinence 6 months after birth & $24(6)$ & $16(4)$ & 1.55 (0.81 to 2.97) & $1.66(0.82$ to 3.37$)$ \\
\hline
\end{tabular}

*Adjusted for recruitment centre only (as a stratification factor).

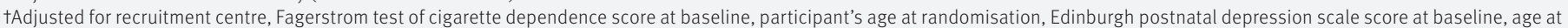
leaving full time education, and partner's smoking status at baseline.

¥Smoked fewer than five cigarettes since quit day.

$\S$ Between 36 weeks' gestation and 10 weeks after birth.

qValidated by exhaled carbon monoxide or salivary cotinine levels, or both; if both measures were available both were required for validation. Biochemical tests did not validate report of not smoking (that is, probable false reporting of cessation) in 12 of 42 women (29\%) in physical activity group and 19 of 44 (43\%) in control group.

${ }^{\star *}$ Biochemical tests did not validate report of not smoking (that is, probable false reporting of cessation) in 4 of 51 women (8\%) in physical activity group and in 7 of 61 (11\%) in control group.

higher and confidence intervals narrower, and the difference in abstinence between treatment and control was minimal even at the upper end of the confidence interval. Thus despite not reaching the target sample size, our results suggest there is unlikely to be a clinically relevant difference. Quit rates were lower than in previous pregnancy trials with less rigorous outcome measures but were similar to those for studies using comparable outcome measures. ${ }^{8}$ For example, two previous UK based large trials of a smoking cessation intervention during pregnancy observed quit rates at the end of pregnancy of $7.6 \%$ and $7.8 \%$ in a group only receiving behavioural support for smoking ${ }^{32} 34$; similarly, we observed a quit rate of $6.4 \%$ for this group.

\section{Interpretation of findings}

Bias in outcome ascertainment is unlikely to explain the findings, as follow-up rates (at 89\%) were equally high in the groups, and for only $6 \%$ of participants who could not be followed-up was it necessary to assume that they had continued to smoke or relapse. Also, the effect size was independent of the influence of missing outcome data. Less than 10\% reported using non-study behavioural support or nicotine replacement therapy, with similar usage in the groups; therefore it is doubtful that this influenced the results. Low attendance may have affected the outcomes; women in the physical activity group attended a median of only four of 14 sessions. However, despite low attendance rates, the counselling on physical activity may still have substantially increased physical activity levels. Low attendance was not explained by the two potentially treatment related adverse events in the physical activity group, and there

\begin{tabular}{|c|c|c|c|}
\hline \multirow[b]{2}{*}{ Visit time } & \multicolumn{2}{|l|}{ EPDS score } & \multirow{2}{*}{$\begin{array}{l}\beta \text { (difference between physical } \\
\text { activity and control group) }(95 \% \mathrm{Cl})^{*}\end{array}$} \\
\hline & Control group & Physical activity group & \\
\hline Baseline & $393,7.7(5.0)$ & $391,7.6(5.3)$ & 0 \\
\hline End of pregnancy & $194,7.2(5.0)$ & $189,8.0(4.9)$ & 1.06 (0.19 to 1.94$)$ \\
\hline 6 month follow-up & $133,6.6(4.7)$ & $146,6.8(4.8)$ & $0.52(-0.45$ to 1.50$)$ \\
\hline
\end{tabular}

Table 4 | Maternal depression. Values are numbers, means (standard deviations) unless stated otherwise

was no indication that the physical activity intervention increased the incidence of adverse events. As is common in smoking cessation trials, the majority of women who failed to quit stopped attending, suggesting that it was failure of quitting that partly led to low attendance. Qualitative data (to be reported elsewhere) based on interviews with participants suggests that low attendance was also influenced by pregnancy related ailments, poor psychological health, and lack of social support.

Evidence for the intervention influencing processes that might aid cessation, such as confidence for quitting, urges to smoke, or withdrawal symptoms was lacking. There was also no evidence for the intervention reducing depression scores; moreover, scores were significantly higher in the physical activity group than control group at the end of pregnancy; although the margin of this difference was small and unlikely to be clinically important. ${ }^{35}{ }^{36}$ A possible explanation for the depression findings, which is consistent with our qualitative data (to be reported elsewhere), relates to the population of interest and the requirements of the intervention. Those in the intervention group were asked to change two health behaviours simultaneously (that is, smoking and physical activity), while also coping with being pregnant and attending multiple treatment sessions. Participants might have been demoralised by finding this difficult to achieve, resulting in marginally higher depression scores at the end of pregnancy. Neither was there evidence that the intervention reduced weight gain. The effects on outcomes for smoking cessation, depression, and weight may have been limited by having an intervention group with an already active lifestyle and that failed to sufficiently raise their physical activity levels, compared with the control group.

It is reassuring that the numbers of adverse events were similar in the two groups, as it suggests that a physical activity intervention is safe and is unlikely to increase these events in pregnant smokers. Moreover, there were $7 \%$ fewer caesarean sections in the physical activity compared with control group. This finding is consistent with a recent meta-analysis that observed a significantly lower risk of caesarean delivery among women undergoing a physical activity intervention 


\begin{tabular}{|c|c|c|c|}
\hline $\begin{array}{l}\text { Subsamples of maternal body } \\
\text { weight }\end{array}$ & $\begin{array}{l}\text { Physical activity } \\
\text { group }\end{array}$ & Control group & $\begin{array}{l}\text { Mean difference } \\
\text { (physical activity- } \\
\text { control) }(95 \% \mathrm{Cl})^{*}\end{array}$ \\
\hline Gestational weight changet: & $n=74$ & $n=66$ & \\
\hline Early pregnancy weight & $68.3(14.4)$ & $70.3(15.6)$ & - \\
\hline End of pregnancy weight & 80.7 (14.9) & $81.4(14.8)$ & $1.08(-1.08$ to 3.23$)$ \\
\hline Postnatal weight retentionf: & $n=63$ & $n=65$ & \\
\hline Early pregnancy weight & $66.1(14.6)$ & $66.2(12.8)$ & - \\
\hline End of pregnancy weight & $70.7(14.1)$ & $71.3(13.4)$ & $-0.11(-2.27$ to 2.49$)$ \\
\hline
\end{tabular}

* Linear regression model with maternal weight change at end of pregnancy as dependent variable, and randomisation groups, early pregnancy weight, and recruitment centre as independent variables.

tWeight measured before birth.

$\neq$ Weight measured after birth between the trial groups (that is, a ceiling effect). There was no evidence to suggest that the treatment effect was influenced by baseline levels of physical activity; although there was insufficient power to detect this interaction and it is possible that the intervention would have shown stronger effects if a more sedentary population had been targeted.

An alternative explanation is that participants over-reported their activity levels. This is supported by the accelerometer data, which shows, as previously observed, ${ }^{39}$ that physical activity levels were far higher by self report than by accelerometer. A limitation is that only 90 participants were monitored by accelerometer. The baseline characteristics of these women were similar to the remainder who did not wear accelerometers. It would have been preferable to assess physical activity using the accelerometer in all participants, but our pilot study showed that most women would not tolerate wearing them and so we used self reported activity for the primary analysis.

Participants could not be blinded to treatment allocation, and the higher self reports of activity in the physical activity group compared with the control group may be biased by knowledge of allocation. This is consistent with the finding that the overestimate of self reported physical activity, versus the accelerometer data, tended to be higher for the physical activity versus control group. Additionally, accelerometer derived physical activity levels were similar in the two trial groups, although when including all moderate-vigorous physical activity (rather than restricting it to bouts of $\geq 10$ minutes) the physical activity group tended to report more physical activity a week than the control group, which suggests that most increases in activity were likely to be in sporadic bouts lasting less than 10 minutes. Thus it remains possible that women in the physical activity group failed to increase their physical

\begin{tabular}{|c|c|c|c|}
\hline Fetal outcomes (singleton births only*) & Physical activity group $(\mathrm{n}=384)$ & Control group $(n=391)$ & Odds ratiot $(95 \% \mathrm{Cl})$ \\
\hline Miscarriage $\ddagger$ & $6 / 383(2)$ & $10 / 389(3)$ & $0.60(0.22$ to 1.67$)$ \\
\hline Stillbirth $\neq$ & $2 / 377(1)$ & $2 / 379(1)$ & $1.01(0.14$ to 7.24$)$ \\
\hline Neonatal death $\ddagger$ & 0 & $1 / 391(0.3)$ & Not calculated \\
\hline Mean (SD) birth weight (g) & $\mathrm{n}=354,3132.4(581.7)$ & $n=359,3146.8(640.0)$ & $-14.50(-104.52$ to 75.52$)$ \\
\hline Mean (SD) gestational age at delivery (weeks) & $\mathrm{n}=356,39.24(2.1)$ & $\mathrm{n}=348,39.26(2.1)$ & $-0.02(-0.36$ to 0.31$)$ \\
\hline Preterm birth§ (<37 weeks’ gestation) & $35 / 356(10)$ & $26 / 348(7)$ & $1.36(0.80$ to 2.31$)$ \\
\hline Low birth weight§ $(<2500 \mathrm{~g})$ & $38 / 353(11)$ & $44 / 359(12)$ & $0.87(0.55$ to 1.38$)$ \\
\hline Admission to neonatal intensive care unit & $27 / 352(8)$ & $36 / 356(10)$ & $0.74(0.44$ to 1.25$)$ \\
\hline Apgar score at 5 minutes $<7$ & $8 / 344(2)$ & $11 / 351(3)$ & $0.74(0.29$ to 1.85$)$ \\
\hline Cord blood arterial $\mathrm{pH}<7$ & $2 / 130(2)$ & $0 / 125$ & Not calculated \\
\hline Congenital abnormalities & $9 / 346(3)$ & $6 / 348(2)$ & $1.43(0.50$ to 4.13$)$ \\
\hline Assisted vaginal delivery & $46 / 357(13)$ & $32 / 359(9)$ & 1.51 (0.94 to 2.43$)$ \\
\hline Caesarean delivery & $76 / 357(21)$ & $103 / 359(29)$ & $0.67(0.48 \text { to } 0.95)^{\star \star}$ \\
\hline \multicolumn{4}{|c|}{$\begin{array}{l}\text { *For all outcomes, } 10 \text { women with twins ( } 8 \text { in physical activity group) were removed from the denominator. When including twin births the findings for birth outcomes were similar. } \\
\text { tOdds ratios and mean differences were adjusted for recruitment centre (as a stratification factor). } \\
\text { ‡These outcomes were defined a priori as serious adverse events. They were not specified as outcomes in the published protocol and therefore are considered as post hoc outcomes. There } \\
\text { were no maternal deaths, and no serious adverse events were judged to be related to the physical activity intervention. The denominator for miscarriage was calculated as the number } \\
\text { randomised minus the number of elective terminations }(n=3) \text { (elective terminations are excluded as they do not have the potential to miscarry). The denominator for stillbirth was calculated as } \\
\text { the number randomised minus the number of miscarriages and elective terminations (elective terminations and miscarriages were excluded as they do not have the potential to result in a } \\
\text { stillbirth). For all other outcomes, the denominator is the number of singleton live births, excluding those births for which outcome data were missing. } \\
\text { §These variables were not specified as outcomes in the protocol and therefore are considered as post hoc outcomes. } \\
\text { १See supplementary table on bmj.com for a list of congenital abnormalities. } \\
\star \star \mathrm{P}=0.023 \text {. }\end{array}$} \\
\hline
\end{tabular}


activity levels compared with the control group, as planned. If this was the case, it is unlikely that such an intervention offered in routine healthcare would raise physical activity levels sufficiently to aid smoking cessation. A less pragmatic and more efficacy oriented trial (for example, with greater incentives, such as financial, to complete the exercise), and with accelerometer measurement in the whole sample, would be needed to establish whether physical activity in itself aids smoking cessation during pregnancy. There was no evidence that change in self reported physical activity was associated with higher quit rates, although this analysis was underpowered and the confidence intervals were wide.

\section{Conclusions}

In this study, evidence that a pragmatic physical activity intervention was effective for aiding smoking cessation was lacking. An efficacy trial involving thrice weekly sessions of vigorous intensity supervised exercise observed a benefit of a physical activity intervention on long term smoking abstinence among non-pregnant women smokers ${ }^{11}$; therefore it remains possible that a more intensive intervention may aid smoking cessation during pregnancy. However, more intensive exercise may be clinically inappropriate in pregnancy, and experience from the trial suggests that uptake of an intensive intervention would be low and would be difficult to integrate into routine healthcare. In addition, the provision of a comprehensive programme of supervised exercise represents a large investment and, to justify such a programme, the effect of the intervention would need to be substantial. We conclude that the reported physical activity programme is unlikely to assist pregnant smokers in stopping smoking. However, recommending physical activity to pregnant women clearly remains indicated for general health during pregnancy.

We thank the following investigators for their contributions: Ory Bolooki (midwife), Janet Brown (midwife), Sarah Cleary (midwife), Caroline Dixon (midwife), Julie Fuller (midwife), Gail Harding (midwife), Maggie Hart (nurse), Tracey Kilbane (research assistant), Rachel Lex (midwife), Zoe Magrath (midwife), Ilia Papachristou (research assistant), Beth Steff (midwife), and Bettina Wanninkhof (midwife). We thank the independent members of the trial steering committee: Iim Thornton (chair), Ann McNeill, Sue Cooper, Kim Watts, and Serena Cox, and we thank Dale Esliger for assistance with analysing and interpreting the accelerometer data.

Contributors: MU, TC, SL, PA, IM, RW, BL, BM, and AT conceived the study, wrote the protocol, and obtained funding. MU managed the day to day running of the trial, including all participant follow-up. MR, SL, and $\mathrm{MU}$ designed the data analysis. $\mathrm{AD}$ advised on the study design and assisted in the design of the analysis for maternal depression and weight. MR conducted all the analyses. All authors had full access to the data, take responsibility for the integrity of the data and the accuracy of the data analysis, contributed to the interpretation of the results, and reviewed and approved the final manuscript. MU drafted the article and is the guarantor. The views and opinions expressed in this article are those of the authors and do not necessarily reflect those of the National Institute for Health Research (NIHR) health technology assessment programme, the NIHR, the National Health Service, or the English Department of Health.

Funding: This study was funded by the NIHR health technology assessment programme (grant 07.01.14). The funder had no role in the design and conduct of the study; collection, management, analysis, and interpretation of the data; preparation, review, or approval of the manuscript; and decision to submit the manuscript for publication.
Competing interests: All authors have completed the ICMJE uniform disclosure form at www.icmje.org/coi disclosure.pdf (available on request from the corresponding author) and declare: no support from any organisation for the submitted work; in the past three years PA has done one day of consultancy for Pfizer concerning general smoking cessation advice and not about particular products, and RW has undertaken research and consultancy for companies (Pfizer and GlaxoSmithKline) that develop and manufacture smoking cessation drugs in the past three years; in the past three years TC has been paid for speaking at two educational events that were part or wholly sponsored by a company (Pierre Fabre Laboratories, France) that manufactures nicotine replacement therapy; RW is an unpaid trustee of the stop smoking charity QUIT and an unpaid director of the National Centre for Smoking Cessation and Training; no other relationships or activities that could appear to have influenced the submitted work

Ethical approval: The research protocol was approved by the Wandsworth Research Ethics Committee.

Data sharing: The guarantor $(\mathrm{MU})$ is willing to examine all requests for the full dataset after a period of three years from the date of this publication. Participants did not give consent for data sharing but the data are anonymised and risk of identification is low.

Transparency: The lead author (MU) affirms that the manuscript is an honest, accurate, and transparent account of the study being reported; that no important aspects of the study have been omitted; and that any discrepancies from the study as planned (and, if relevant, registered) have been explained.

This is an Open Access article distributed in accordance with the terms of the Creative Commons Attribution (CC BY 4.0) license, which permits others to distribute, remix, adapt and build upon this work, for commercial use, provided the original work is properly cited. See: http://creativecommons.org/licenses/by-nc/4.0/.

$1 \quad$ Kallen $\mathrm{K}$. The impact of maternal smoking during pregnancy on delivery outcome. Eur I Public Health 2001;11:329-33.

2 Rogers JM. Tobacco and pregnancy. Reprod Toxicol 2009;28:152-60.

3 Salihu HM, Wilson RE. Epidemiology of prenatal smoking and perinatal outcomes. Early Hum Dev 2007:83:713-20.

4 NHS Information Centre. Infant feeding survey 2010: early results. NHS Information Centre for Health and Social Care, 2011.

5 Tong VT, Dietz PM, Morrow B, et al. Trends in smoking before, during and after pregnancy-pregnancy risk assessment monitoring system, United States, 40 sites, 2000-2010. MMWR Surveill Summ 2013;62:1-19.

6 Al-Sahab B, Saqib M, Hauser G, et al. Prevalence of smoking during pregnancy and associated risk factors among Canadian women: a national survey. BMC Pregnancy Childbirth 2010;10:24.

Richmond R. You've come a long way baby: women and the tobacco epidemic. Addiction 2003;98:553-7.

8 Chamberlain C, O'Mara-Eves A, Oliver S, et al. Psychosocial interventions for supporting women to stop smoking in pregnancy. Cochrane Database Syst Rev 2013;10:CD001055.

9 Coleman T, Chamberlain C, Davey MA, et al. Pharmacological interventions for promoting smoking cessation during pregnancy. Cochrane Database Syst Rev 2012·9:CD010078.

10 Haasova, M, Warren FC, Ussher M, et al. The acute effects of physical activity on cigarette cravings: systematic review and meta-analysis with individual participant data (IPD). Addiction 2012;108:26-37.

11 Marcus BH, Albrecht AE, King TK, et al. The efficacy of exercise as an aid for smoking cessation in women: a randomised controlled trial. Arch Intern Med 1999;159:1229-34.

12 Ussher MH, Taylor A, Faulkner G. Exercise interventions for smoking cessation. Cochrane Database Syst Rev 2012;1:CD002295.

13 Garber CE, Blissmer B, Deschenes MR, et al. American College of Sports Medicine. American College of Sports Medicine position stand. Quantity and quality of exercise for developing and maintaining cardiorespiratory, musculoskeletal, and neuromotor fitness in apparently healthy adults: guidance for prescribing exercise. Med Sci Sports Exerc 2011;43:1334-59

14 Ussher M, Aveyard P, Coleman T, et al. Physical activity as an aid to smoking cessation during pregnancy: two feasibility studies. BMC Public Health 2008;8:328.

15 Ussher M, Aveyard P, Manyonda I, et al. Physical activity as an aid to smoking cessation during pregnancy (LEAP) trial: study protocol for a randomized controlled trial. Trials 2012;13:186.

16 Michie S, Hyder N, Walia A, et al. Development of a taxonomy of behaviour change techniques used in individual behavioural support for smoking cessation. Addict Behav 2011:36:315-9.

17 Heatherton T, Kozlowski L, Frecker T, et al. The Fagerström test for nicotine dependence: a revision of the Fagerström tolerance questionnaire. Br J Addict 1991;86:1119-27. 
18 Blair SN, Haskell WL, Ho P, et al. Assessment of habitual physical activity by seven-day recall in a community survey and controlled experiments. Am J Epidemiol 1985;122:794-804

19 Murray D, CoxJL. Screening for depression during pregnancy with the Edinburgh Depression Scale (EPDS). I Reprod Infant Psychol 1990;8:99-107.

20 Marcus BH, Selby VC, Niaura RS, et al. Self-efficacy and the stages of exercise behavior change. Res Q Exerc Sport 1992;63:60-6

21 West R, Willis N. Double-blind placebo controlled trial of dextrose tablets and nicotine patch in smoking cessation. Psychopharmacology 1998;136:201-4.

22 Babor TF, de la Fuente JR, Saunders J, et al. AUDIT: the Alcohol Use Disorders Identification Test-guidelines for use in primary health care. World Health Organization, 1992.

23 West R, Russell M. Pre-abstinence smoke intake and smoking motivation as predictors of severity of cigarette withdrawal symptoms. Psychopharmacology 1985;87:334-6.

24 Ussher M, Beard E, Abikoye G, et al. Urge to smoke over 52 weeks of abstinence. Psychopharmacology 2013;226:83-9.

25 West R, Hajek P, Stead L, Stapleton J. Outcome criteria in smoking cessation trials: proposal for a common standard. Addiction 2005;100:299-303.

26 Lumley J, Chamberlain C, Dowswell T, et al. Interventions for promoting smoking cessation during pregnancy. Cochrane Database Syst Rev 2009;3:CD001055.

27 Chamberlain C, O'Mara-Eves A, Oliver S, et al. Psychosocial interventions for supporting women to stop smoking in pregnancy. Cochrane Database Syst Rev 2013;10:CD001055.

28 Hedeker D, Mermelstein RJ, Demirtas H. Analysis of binary outcomes with missing data: missing=smoking, last observation carried forward, and a little multiple imputation. Addiction 2007;102:1564-73.

29 Troiano RP, Berrigan D, Dodd KW, et al. Physical activity in the United States measured by accelerometer. Med Sci Sports Exerc 2008;40:181-8.
30 Gates S, Brocklehurst P. How should randomised trials including multiple pregnancies be analysed? BJOG 2004;111:213-9.

31 Fergusson D, Aaron SD, Guyatt G, Hébert P. Post-randomisation exclusions: the intention to treat principle and excluding patients from analysis. BMJ 2002;325:652-4.

32 Coleman T, Cooper S, Thornton JG, et al. Smoking, Nicotine, and Pregnancy (SNAP) trial team. A randomized trial of nicotine-replacement therapy patches in pregnancy. N Engl J Med 2012;366:808-18.

33 Gavin NI, Gaynes BN, Lohr KN, et al. Perinatal depression: a systematic review of prevalence and incidence. Obstet Gynecol 2005; 106:1071-83.

34 Hajek P, West R, Lee A, et al. Randomized controlled trial of a midwife-delivered brief smoking cessation intervention in pregnancy. Addiction 2001;96:485-94

35 Affonso DD, De AK, Horowitz JA, et al. An international study exploring levels of postpartum depressive symptomatology. J Psychosom Res 2000;49:207-16.

36 Matthey S. Calculating clinically significant change in postnata depression studies using the Edinburgh Postnatal Depression Scale. J Affect Disord 2004;78:269-72.

37 Domenjoz I, Kayser B, Boulvain M. Effect of physical activity during pregnancy on mode of delivery. Am J Obstet Gynecol 2014;211:401 (e1-11)

38 Ussher M, Ah-Yoon M, West R, et al. Factors associated with exercise participation and attitudes to exercise among pregnant smokers. J Smok Cessat 2007;2:12-6.

39 Harrison CL, Thompson RG, Teede HJ, et al. Measuring physical activity during pregnancy. Int J Behav Nutr Phys Act 2011;8:19.

(C) BMJ Publishing Group Ltd 2015

Additional material

Table of adverse events and list of less common adverse events 